\title{
Numerical analysis of resonant multipolar instabilities in high power Klystrons
}

\author{
J.C. Cai, I. Syratchev, G. Burt
}

\begin{abstract}
The monopole monotron instability is a well-known phenomenon in the linear beam devices. In recent development of a high efficiency $50 \mathrm{MW}$ X-band klystron such instabilities were found and mitigated in a special $2^{\text {nd }}$ harmonic multi-cell cavities triplet that is used to improve the klystron performance. In further simulations of the klystron, using 3D Particle-In-cell computer code, the more rare and complicated phenomenon, previously unreported in non-relativistic devices, associated with rotating multipolar monotron instabilities was discovered. In this letter, the qualitative and quantitative analysis of these oscillations will be presented. Various resonant multipolar instabilities suppression strategies will be introduced and discussed in detail.
\end{abstract}

Index Terms-Hybrid modes, Instability, Klystron.

\section{INTRODUCTION}

$\mathrm{K}$ LYSTRONS are linear beam devices, where the electron beam interacts with an array of the bunching cavities. In this process, the initial velocity modulation of the particles is converted into a beam current RF modulation [1,2]. In some cases, at high frequencies, to improve the bunching quality a few coupled cells are used instead of a single cavity to enhance the impedance $[3,4]$. Another important measure that allows the improvement of the klystron efficiency and the reduction of the RF bunching circuit length is the use of harmonic cavities $[5,6]$. A multicell second harmonic RF circuit was implemented through the design of $12 \mathrm{GHz}, 50 \mathrm{MW}$ high efficiency klystron [7,8]. To accommodate a $400 \mathrm{kV}, 190 \mathrm{~A}$ beam current, the required beam tunnel aperture in this tube is rather large with respect to the second harmonic wavelength. Through optimization it was concluded that three coupled cells (triplet) operating in the $\pi$ mode provided sufficient impedance, beam coupling and mode separation. The triplet geometry is shown in Fig. 1. However, with large beam power, such a topology appeared to be sensitive to spurious mode oscillations. The possibility of monotron oscillations [9-11] in this tube were investigated in detail and mitigated by adjusting the length of the triplet period [12]. The threshold current of the monopolar monotron oscillation only weakly depends on the magnetic field since longitudinal beam-wave interaction dominates the process. Therefore, in this study, in order to compare the monotron oscillations simulation results provided by different computer tools, we initially used a large external magnetic field $\left(\mathrm{B}_{\mathrm{z}}=15 \times \mathrm{B}_{\text {Brillouin }}\right)$ to freeze the beam transversely [13]. Surprisingly when the external magnetic field in this immersive magnetic system was later reduced to the operating value of

Jinchi Cai (jinchi.cai@cern.ch) and Graeme Burt

(graeme.burt@cockcroft.ac.uk) are with Department of Engineering of

Lancaster University, LA1 4YW, Lancaster, United Kingdom; Igor

Syratchev (igor.syratchev@cern.ch) is with BE/RF group at CERN

(European Organization for Nuclear Research), 1211, Geneva 23,

Switzerland.
$0.35 \mathrm{~T}\left(2.2 \times \mathrm{B}_{\text {Brillouin }}\right)$ in dedicated Particle-In-Cell (PIC) long pulse simulations (2000 ns), using CST/3D [14], a rare and complicated instability evolved. This time we observed an unusual situation, where the dc beam is coupled to the rotating multipolar hybrid $\mathrm{HE}$ modes $\left(\mathrm{TE}_{21}\right.$ and $\mathrm{TE}_{31}$-like) in the triplet rather than $\mathrm{TM}_{0 \mathrm{n}}$-like monopolar modes. The onset time for these oscillations was between $500 \mathrm{~ns}$ and $1000 \mathrm{~ns}$, thus we could not spot such problems in the earlier klystron simulations when applying rather short (200-300ns) simulation times. Such phenomenon associated with an azimuthally asymmetric mode was previously discovered experimentally in relativistic vacuum electron devices [15]. Follow-up studies are either directly based on 3D PIC simulation or qualitatively treated it as a longitudinal interaction process [16,17]. In non-relativistic traditional Klystron with MW output power level, such a phenomenon has never been reported before. The only similarity we have found in the literature is a beam transverse kick effect originated by the $\mathrm{TE}_{11}$-like mode trapped in the drift tube between the bunching cavities [18-20]. In some other publications $[21,22]$ it was suggested that using stainless steel instead of copper for the drift tubes could prevent the klystron from oscillating in an unspecified trapped High Order mode (HOM).

A deeper understanding of the mechanisms behind the beamwave coupling and finding mitigation methods of the multipolar beam instability requires massive 3D PIC simulations. Such simulations are very time consuming. For example, long-pulse (2000ns) PIC simulations using a computer cluster with GPU accelerators (Kepler/Maxwell Type card supported by CST) takes about 2 days ( 0.5 million mesh cells). In this letter, we will first give a qualitative analysis of the resonant multipolar instabilities. Next, we will explain an innovative method for the fast analysis of these instabilities using a beam loading calculation in 3D PIC simulations with imported eigen fields of the modes. Comparing with other analytical/numerical methods for beam loading analysis of 3D modes, the methods put forward in this paper are more universal and convenient to execute $[23,24]$. Finally, we will discuss the different methods

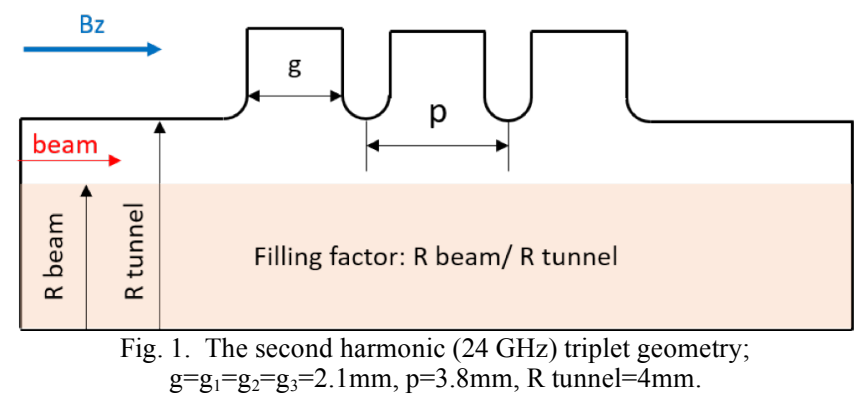


which allow suppression of the multipolar oscillations.

\section{RESONANT MULTIPOLAR INSTABILITIES ANALYSIS}

The resonant multipolar instabilities in the second harmonic triplet (see Fig.1) made of copper with conductivity of $5.8 \times 10^{7}$ $\Omega^{-1} \mathrm{~m}^{-1}$ were originally observed in $3 \mathrm{D}$ PIC simulation. In this simulation an idealized dc beam $(400 \mathrm{kV}, 190$ A) with an emission radius of $2.8 \mathrm{~mm}$ (filling factor 0.7 ) and only axial momentum was injected from a metal surface at the end of the beam tunnel, to include space-charge depression and imitate the practical cylindrical beam with little azimuthal rotation and
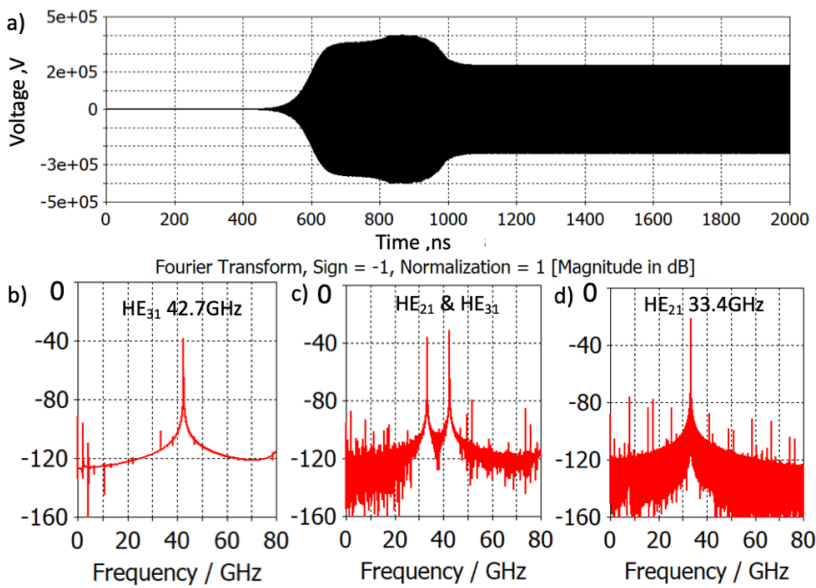

Fig. 2. a) Voltage signal measured by the voltage monitor in the first gap. b) spectrum of this signal before $700 \mathrm{~ns}$. c) spectrum of this signal between $700 \mathrm{~ns}$ and $1100 \mathrm{~ns}$. d) spectrum of this signal after $1100 \mathrm{~ns}$.

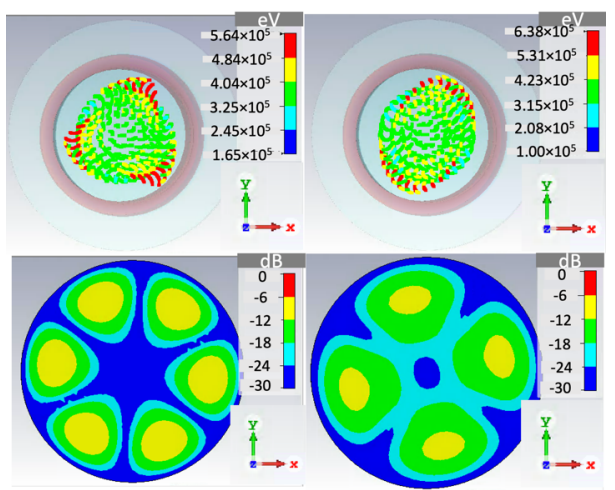

Fig. 3. Particles distributions (top, energy scaled by color) and electric field plots (bottom, field amplitude normalized in $\mathrm{dB}$ scaled by color) at the triplet cross section for $\mathrm{HE}_{31}$ (left) and for $\mathrm{HE}_{21}$ (right) oscillations.

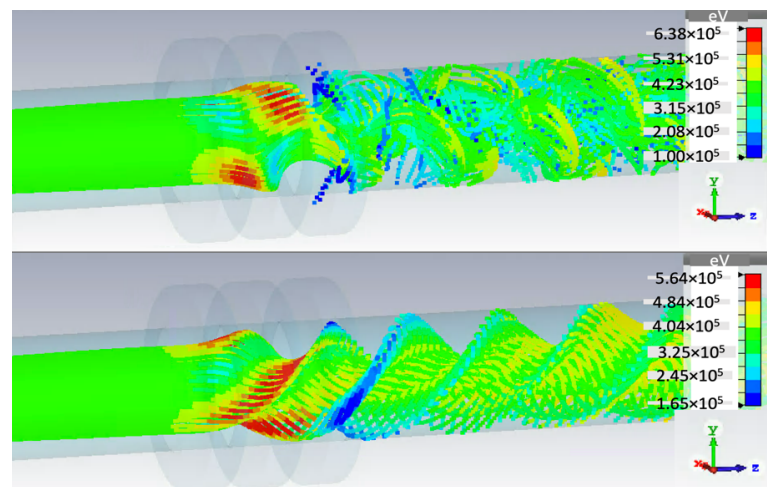

Fig. 4. 3D views of the particles distributions for $\mathrm{HE}_{21}$ oscillation (top) and $\mathrm{HE}_{31}$ oscillation (bottom). Electron energy scaled by color. evenly distributed current density. The external magnetic solenoidal field with an amplitude of $0.35 \mathrm{~T}$ was used in this simulation to confine the beam. After 500 ns we observed the onset of an instability by measuring the amplitude of the electric field with a voltage monitor located in the first gap of the triplet, which saturated in about 150 ns. After 900 ns the oscillation changed its amplitude, saturating at a new level, suggesting the involvement of multiple modes or instabilities. The evolution in time of the envelope of the measured oscillation and its corresponding spectrum is shown in Fig. 2. By analyzing the electric field and beam patterns at the triplet cross section (see Fig. 3) and the signal spectrum, we concluded that initially the instability is associated with the rotating $\mathrm{TE}_{31}$-like hybrid mode $\left(\mathrm{HE}_{31}\right)$ at $42.7 \mathrm{GHz}$ and after $700 \mathrm{~ns}$, the rotating $\mathrm{TE}_{21}$-like hybrid mode $\left(\mathrm{HE}_{21}\right)$ at $33.4 \mathrm{GHz}$ involves in which finally becomes dominant after 1100ns. This conclusion was confirmed by simulating the modes in the triplet using CST eigenmode solver and matching the eigen frequencies with the instability frequencies. The rotation directions of the modes were opposite from each other: $\mathrm{HE}_{31}$ was rotating clockwise $(+)$ and $\mathrm{HE}_{21}$ was rotating contra clockwise (-). Flipping the direction of external magnetic field, the modes rotation was also alternated, whilst the oscillation amplitude was not changed. More details are shown in Fig. 4. One may see, that in interaction with these modes, electron beam receives an azimuthal modulation, and the spiral trajectories appear like a rotating Archimedes' screw. With increasing magnetic field, the $\mathrm{HE}_{21}$ oscillation disappears when $\mathrm{B}_{\mathrm{z}}$ exceeds $0.45 \mathrm{~T}$ and after $0.70 \mathrm{~T}$ the $\mathrm{HE}_{31}$ mode oscillation vanishes as well. The oscillations frequencies nearly do not depend on the magnetic field amplitude and/or direction. In steady state the surface electric field approaches very high values, 480MV/m $\left(\mathrm{HE}_{21}\right)$ and $360 \mathrm{MV} / \mathrm{m}\left(\mathrm{HE}_{31}\right)$. With such behavior, we can classify these instabilities as monotron type instabilities with multipolar modes. It should be noted that this instability does not occur with monopolar modes as these modes cannot rotate. Here we introduce the Beam Loading quality factor $\left(Q_{\text {beam }}\right)$, which describes the ratio of the stored energy in the resonant mode to the power exchange between the electron beam and the resonant mode in small signal region with DC beam propagated through the cavity gap [25-27]. Unlike monopole modes, $Q_{\text {beam }}$ here depends not only on the beam current, but also is a nonlinear function of the external magnetic field and the beam filling factor. From monotron oscillation theory it follows, that an oscillation can be established if $Q_{\text {beam }}$ is negative and its absolute value is smaller the value of the mode intrinsic quality factor $\left(Q_{i n}\right)$. To calculate $Q_{\text {beam }}$ from the PIC simulations, we can use the exponential growth of the oscillation shortly after the onset and pick up two voltage amplitudes $\left(V_{1}, V_{2}\right)$ at two different moments of time $\left(t_{1}, t_{2}\right)$ :

$$
Q_{\Sigma}=\frac{\pi f}{\ln \left(V_{1} / V_{2}\right)}\left(t_{2}-t_{1}\right)
$$

here $f$ is the mode frequency. With known value of the mode $Q_{\text {in }}$ and calculated value of $Q_{\Sigma}, Q_{\text {beam }}$ can be reconstructed:

$$
\frac{1}{Q_{\Sigma}}=\frac{1}{Q_{\text {in }}}+\frac{1}{Q_{\text {beam }}}
$$

This straightforward method of $Q_{\text {beam }}$ calculation still requires a long PIC simulation time. Also, it is impossible to disentangle 
any other modes where $Q_{\text {beam }}$ is larger. A more efficient approach to calculate $Q_{\text {beam }}$ was extrapolated from the method used in [12]. It is related to the monotron small signal theory, when beam dynamic calculations (BDC) in PIC can be performed with known (imported from CST eigen solver) eigen field map of the mode. The mode rotation can be imposed in the simulation by using two identical field maps with an RF phase shift of +/-90 degrees between them, with the sign controlling the rotation direction. With this method, one does not need to use the full geometry in the simulation, but only the smooth beam pipe region. To perform the $Q_{\text {beam }}$ calculation it is sufficient to measure the beam power at the collision plane $\left(P_{\text {end }}\right)$ for two regimes, the first without the imported mode field map $(d c)$ and the second with the imported mode field map $(r f)$ :

$$
Q_{\text {beam }}=\frac{\omega W}{\Delta P}=\frac{2 \pi f W}{P_{\text {end,rf }}-P_{\text {end, } d c}}
$$

here $f$ is the frequency of the mode and $W$ is the stored energy. To obey the small signal regime, the system shall be linear, thus $W$ shall be set small enough. This condition can be calibrated by measuring if $P_{e n d, r f}$ changes linearly with changing $W$. Such a method can be shown to be reliable when calculating $Q_{\text {beam }}<1 \times 10^{5}$ and is suggested as a general approach for the evaluation of various monotron instabilities using fast (few

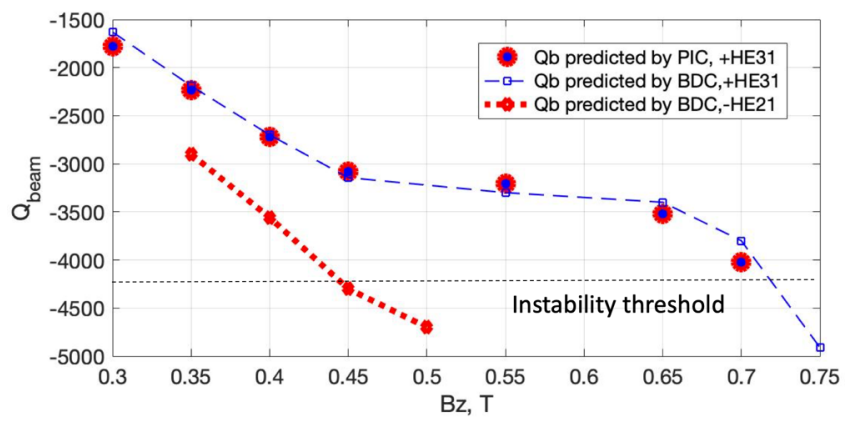

Fig. 5. $Q_{\text {beam }}$ as function of the magnetic field for $\mathrm{HE}_{31}$ and $\mathrm{HE}_{21}$ modes; $Q_{\text {in }}$ is approximately 4200 for both modes.

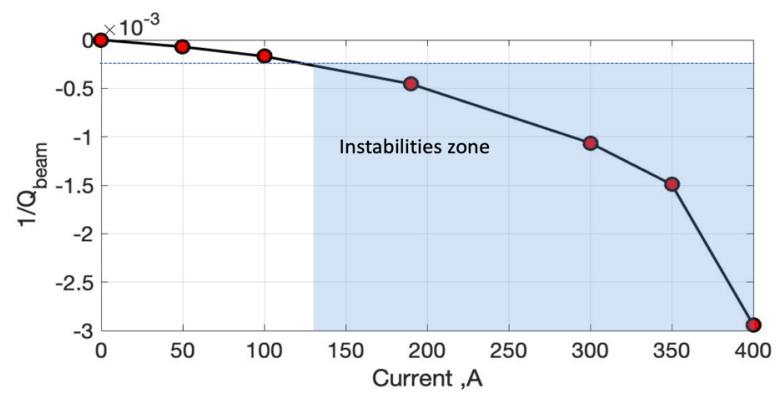

Fig. 6. $Q_{\text {beam }}$ as the function of the beam current for the dominant $\mathrm{HE}_{31}$ mode $\left(\mathrm{B}_{\mathrm{z}}=0.35 \mathrm{~T}\right)$.

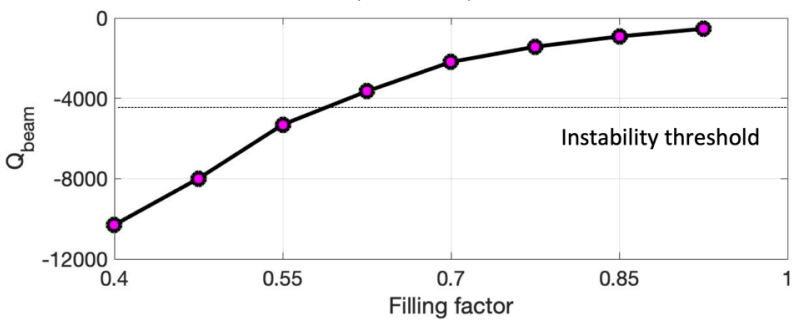

Fig. 7. $Q_{\text {beam }}$ as a function of the beam filling factor for the dominant $\mathrm{HE}_{31}$ mode $\left(\mathrm{B}_{2}=0.35 \mathrm{~T}\right)$. minutes on the same sever mentioned before), almost steady state 3D PIC simulations. More specifically, the stabilization time for beam dynamics calculations (BDC) conducted in CST PIC simulation should be longer than the current rise time ( $\sim 1 \mathrm{~ns})$ plus several electron flight times ( $\sim 1 \mathrm{~ns})$, therefore several ns will be enough rather than $1000 \mathrm{~ns}$ in a full PIC simulation. Such BDC consider all effects, like relativistic effects, space charge effects, 3D particle trajectories, etc. Using BDC, we have calculated $Q_{\text {beam }}$ for the modes, which rotate in opposite directions compared to the ones observed in simulations. Both showed positive $Q_{\text {beam }}$ values (several thousand) within the range of external magnetic field between $0.3 \mathrm{~T}$ and $0.9 \mathrm{~T}$. Which is consistent with observations made in the direct PIC simulations. In Fig. 5 the direct calculation of $Q_{\text {beam }}$ (Eq. 1) is compared with BDC calculation. Direct calculations for $\mathrm{HE}_{21}$ mode were not possible, because $Q_{\text {beam }}$ of this mode is larger than $Q_{\text {beam }}$ of $\mathrm{HE}_{31}$ mode. Thus, only $\mathrm{BDC}$ results of $\mathrm{HE}_{31}$ mode are shown. However, BDC predictions are consistent with observation made in PIC simulation, when $\mathrm{HE}_{21}$ oscillation disappeared with $\mathrm{B}_{\mathrm{z}}$ exceeding $0.45 \mathrm{~T}$.

Unlike the monotron oscillations of the monopole modes, where the threshold current is inversely proportional to $Q_{\text {beam }}$, for the multipolar oscillations this correlation is more complicated, see Fig. 6. From this observed relationship between $\mathrm{Q}_{\text {beam }}$ and magnetic field, we can conclude that transverse beam-wave interaction plays an important role for this type of oscillation. In this case, the particles trajectories transverse component is greatly affected by the beam current itself, especially when the confining magnetic field is close to the Brillouin magnetic field. Another peculiarity of the multipolar instability is the $Q_{\text {beam }}$ dependence on the beam radius (filling factor), see Fig. 7. In these illustrations, for simplicity, we fixed the beam voltage $(400 \mathrm{kV})$ and the external magnetic field, disregarding the fact that with changing the current and/or the beam radius, the confining magnetic field might need to be re-adjusted.

\section{RESONANT MULTIPOLAR INSTABILITIES SUPPRESSION STRATEGIES}

Following the results shown in Fig. 5, the increase of the external magnetic field from $0.35 \mathrm{~T}$ to $0.7 \mathrm{~T}$ will eliminate the resonant multipolar instabilities. It is also straightforward that with increasing the magnetic field, the beam filling factor can be reduced to below 0.55 , thus the klystron stability will be fully assured. However, this approach is not economically efficient, as the solenoid cost and power consumption will be increased significantly.

Another solution is to use stainless steel for the triplet fabrication (conductivity $1.3 \times 10^{6} \Omega^{-1} \mathrm{~m}^{-1}$ ) instead of the copper (conductivity $\sim 5.8 \times 10^{7} \Omega^{-1} \mathrm{~m}^{-1}$ ). In this case the $Q_{i n}$ of each of the modes will be reduced by a factor of 7 , which is proportional to the square root of the conductivity of the metallic wall for a given mode [28]. That is more than sufficient to eliminate both oscillations, see Fig. 5. As a drawback, such an approach could introduce additional complication and cost increases into the tube fabrication process.

A more rewarding strategy is to review the geometry of the 
triplets itself. In our previous studies, monopole monotron oscillations in the triplet were removed by adjusting the cells period length to $3.8 \mathrm{~mm}$ [12]. We have tried the same technique in attempt to mitigate the multipolar oscillations, but this attempt was not successful due to the number of modes involved, see Fig. 8.

Next, we have studied the HOM modes frequency detuning method. The cells period length was slightly tapered, whilst keeping the overall length and iris thickness constant. Meaning that central cell was not modified. In this exercise, the outer cells radii were adjusted to preserve the $\mathrm{TM}_{01}$ resonant frequency of the individual cells. Such modification practically did not affect the effective impedance of the operating $24 \mathrm{GHz}$ $\pi$ mode. This approach was rather successful, when the period

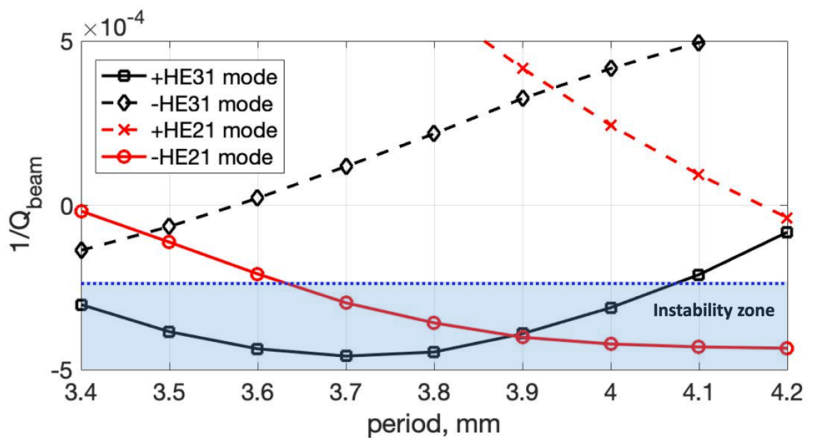

Fig. 8. $Q_{\text {beam }}$ as a function of cell period for multipolar modes in the triplet.

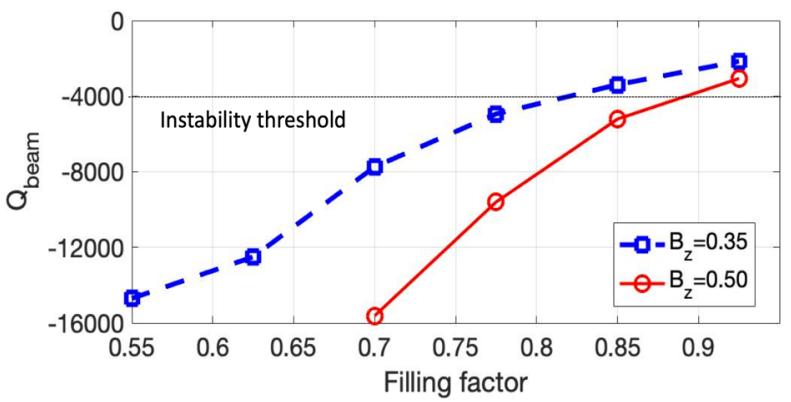

Fig. 9. $Q_{\text {beam }}$ of $\mathrm{HE}_{21}$ mode as a function the filling factor for two values of the confining magnetic field in the modified triplet.

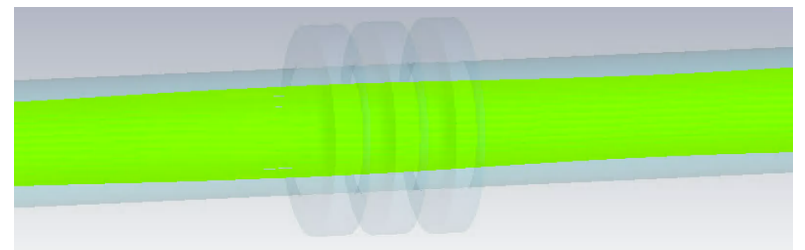

Fig. 10. Particle trajectories after 2000ns PIC simulations in the modified triplet.

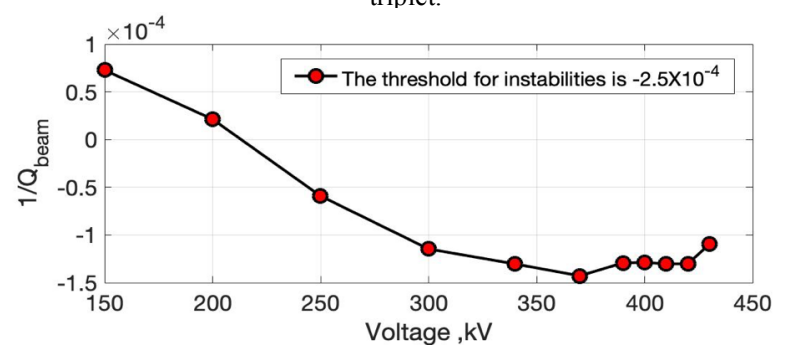

Fig. 11. $Q_{\text {beam }}$ of $\mathrm{HE}_{21}$ mode simulated for the range of beam voltages from $150 \mathrm{kV}$ to $430 \mathrm{kV}$ with fixed beam perveance $\left(\mathrm{B}_{\mathrm{z}}=0.35 \mathrm{~T}\right)$. length of the two extreme cells was changed by $0.2 \mathrm{~mm}$. The dominant $\mathrm{HE}_{31}$ mode was fully eliminated, with $Q_{\text {beam }}$ being positive for both rotating polarizations. The remaining $\mathrm{HE}_{21}$ mode with original rotating polarization survived, but the negative value of $Q_{\text {beam }}$ was increased from 2900 to 8000, thus the triplet was moved out of the instability zone. $Q_{\text {beam }}$ dependence on the filling factor for two values of the confining magnetic field are shown in Fig. 9. From these results, we can conclude that with the designed value of the filling factor $(0.7)$ and expected beam scalloping in the drift tube below $10 \%$, the klystron will be stable when operated with the nominal amplitude of confining magnetic field of 0.35T. Particle trajectories for this operating point after 2000ns PIC simulations are shown in Fig. 10. The modified triplet stability was studied for the range of the beam voltages from $150 \mathrm{kV}$ to $430 \mathrm{kV}$ with fixed beam perveance $\left(0.75 \mu \mathrm{A} / \mathrm{V}^{3 / 2}\right)$ and $0.35 \mathrm{~T}$ external magnetic field, see Fig. 11. In these simulations, the $Q_{\text {beam }}$ value remains sufficiently above (factor 1.7 ) the threshold limit for the entire range of simulated voltages.

It is noteworthy that the example presented here is operated in an immersive magnetic system, meaning that the azimuthal velocity of the electron beam is theoretically zero at the entrance of the RF circuit and very small when the beam is passing through the circuit in the DC scenario. However, the idealized emission model could be modified to handle error analysis when the magnetic flux is not well aligned with the beam trajectory in the gun area. This is equivalent to putting less/more $\mathrm{B}_{\mathrm{z}}$ field on the emission plate than in the latter RF circuit in our model to rotate the beam in purpose. The nonuniform $\mathrm{B}_{z}(\mathrm{z})$ with an abrupt field change just after the emission plate shows that the $\mathrm{Q}_{\text {beam }}$ of the $\mathrm{HE}_{21}$ mode of the modified triplet with the same filling factor $(0.7)$ and saturated $\mathrm{B}_{\mathrm{z}}$ field $(0.35 \mathrm{~T})$ will be affected by about $20 \%$ if the variation of $\mathrm{B}_{\mathrm{z}}$ field on the emission plate is $10 \%$. Such a sensitivity study is further proof that the transverse beam wave interaction is a nonnegligible factor for this type of instability. More realistic or a more general (optics from shield/partially shield gun) model could be established with the import of emission information from beam optics simulations, where the technique proposed in this paper will still apply for resonant multipolar instability analysis regardless of the specific optics situations.

In klystron amplifiers, any spurious oscillation can abruptly interrupt the RF power generation and must be eliminated at the design stage. However, there is a class of RF generators, which use the monopole monotron instabilities and $\mathrm{dc}$ beam to generate coherent microwave radiation [29]. It is proposed here for further study, that resonant multipolar instabilities can be also used for the megawatt level RF power generation at high frequencies $(100 \mathrm{GHz})$, profiting from the reasonable level of the confining magnetic field and large beam tunnel aperture to accommodate large current.

\section{CONCLUSION}

The rare phenomenon of multipolar beam instabilities in the second harmonic $(24 \mathrm{GHz})$ cavities triplet of the $50 \mathrm{MW}, 12$ $\mathrm{GHz}$ high efficiency klystron was observed in 3D PIC simulation. The numerical analysis of the instabilities allowed 
us to classify these oscillations as monotron type instabilities associated with a rotating $\mathrm{TE}_{31}$-like hybrid mode $\left(\mathrm{HE}_{31}\right)$ at $42.7 \mathrm{GHz}$ and a $\mathrm{TE}_{21}$-like hybrid mode $\left(\mathrm{HE}_{21}\right)$ at $33.4 \mathrm{GHz}$. To make quantitative analysis of these oscillations, a novel technique of beam loading quality factor calculation using fast PIC simulations was proposed. Using this method, the impact of the various parameters like beam current and voltage, amplitude of the external magnetic field and beam filling factor on the instabilities onsets was studied. Resonant multipolar instabilities suppression strategies were presented and discussed in detail. The most economically and technologically efficient method, the triplet HOM detuning, was demonstrated to be effective to ensure klystron operation far from the instability region. Finally, the modified triplet could work stably with a filling factor of 0.7 and a magnetic field of $0.35 \mathrm{~T}$ in such a high-power Klystron without any oscillation.

\section{REFERENCES}

[1] R.K. Parker, R.H. Abrams, B.G. Danly, B. Levush "Vacuum electronics", IEEE Trans. On Microwave Theory and Techniques, vol.50 no.3, pp.835845, March 2002, doi: 10.1109/22.989967.

[2] D.K. Abe, D.E. Pershing, K.T. Nguyen, F.N. Wood, R.E. Myers, E.L. Eisen, M. Cusick, and B. Levush. "Demonstration of an S-band, 600-kW fundamental-mode multiple-beam Klystrons," IEEE Electron Device Letters, vol. 26, no. 8, pp. 590-592, Aug. 2005, doi: 10.1109/LED.2005.852540.

[3] J. Ju, J. Zhang, T. Shu, and H. Zhong, "An improved X-band triaxial Klystron amplifer for gigawatt long-pulse high-power microwave generation", IEEE Electron Device Letters, vol. 38, no. 2, pp. 270-272, Feb. 2017, doi: 10.1109/LED.2016.2646679.

[4] X. Wang, S. Li, X. Zhang, S. Jiang, Z Wang, H. Gong, Y. Gong, B. Basu and Z. Duan, "Novel S-Band Metamaterial Extended Interaction Klystron", IEEE Electron Device Letters, vol. 41, no. 10, pp. 1580-1582, October. 2020, doi: 10.1109/LED.2020.3015036.

[5] E. L. Lien, "High efficiency klystrons amplifiers", Proc. of the Eights Conference on Microwave and Optical Generation and Amplification, Amsterdam. The Netherlands, 1970.

[6] V. Hill, G. Burt, D. Constable, C. Lingwood, C. Marrelli, I. Syratchev, "Particle-in-cell Simulation of second and third harmonic cavity klystron", in IEEE International Vacuum Electronics Conference, IVEC 2017, London, UK, April 2017, doi: 10.1109/IVEC.2017.8289626.

[7] I. Syratchev, J.C. Cai, "High efficiency Klystron development", CLIC Mini Week 2020, CERN, Geneva, Switzerland. Available: https://indico.cern.ch/event/952778/contributions/4013794/.

[8] Claude Van Daele, "X-band klystrons activities at CPI", Second CompactLight Meeting, Athens, Greece, January 2020. Available: https://indico.cern.ch/event/867582/contributions/3709639/

[9] J.C. Cai, I. Syratchev, "KlyC: 1.5D Large Signal Simulation Code for Klystrons", in IEEE Trans. on Plasma Science, vol.47, no.4, pp.17341741, April 2019, doi: 10.1109/TPS.2019.2904125.

[10] E.J. Graig, "The beam-loading admittance of gridless klystron gaps", IEEE Trans. on Electron devices, vol.145, no.4, pp.273-278, May 1967, doi: 10.1109/T-ED.1967.15942.

[11] E.J. Craig. "Relativistic beam-loading admittance", IEEE Transactions on Electron Devices, Vol. 16, no. 1, pp. 139-139, 1969, doi: 10.1109/TED.1969.16575.

[12] J.C. Cai, I. Syratchev and G. Burt. "Accurate Modeling of Monotron Oscillations in Small-and Large-Signal regimes", IEEE Transactions on Electron Devices, Vol. 67, no. 4, pp. 1797-1803, April 2020, doi: 10.1109/T-ED.2020.2971279.

[13] J. R. Pierce, "Theory and design of electron beams", Toronto: D.Van Nostrand Co., Inc., Jan, 1954.

[14] CST 2018. Available at: https://www.cst.com/products.

[15] X.J. Ge, H.H. Zhong, B.L. Qian, J. Zhang, L. Liu, L. Gao, C.W. Yuan and J.T. He. "Asymmetric-mode competition in a relativistic backward wave oscillator with a coaxial slow-wave structure", Appl. Phys. Lett., Vol. 97, no. 24, pp. 241501, December 2010, doi: 10.1063/1.3526726.

[16] D. Zhang, J. Zhang, H.H. Zhong, Z.X. Jin and J.C. Ju. "Asymmetric mode decomposition in an overmoded relativistic backward wave oscillator",
Phys. Plasmas,, Vol. 21, no. 9, pp. 093102, September 2014, doi: 10.1063/1.4894480.

[17] Z. Qi, J. Zhang, Y.J. Xie, Yi. Zhang, Z.H. Wang, X.F. Zhou, J.H. Zhu, Y.Y.Zi and H.H. Zhong. "Analysis on the mechanism of pulse shortening in an X-band triaxial Klystron amplifier due to the asymmetric mode competition”, Phys. Plasmas,, Vol. 23, no. 12, pp. 123103, December 2016, doi: 10.1063/1.4969079.

[18] A. E. Vlieks. "X-band Klystron development at SLAC", SLAC-PUB13741, July 2009.

[19] A. E. Vlieks, "X-band Klystron development at SLAC", X-band RF structure \& Beam Dynamics Workshop. Cockcroft Institute, UK, December 2008.

[20] R. Kowalczyk, A. Haase, E. Jongewaard, M. Kemp, J. Neilson and A. Jensen. "Test of a BAC Klystron", SLAC-PUB-17102, August 2017.

[21] D. Sprehn, R.M. Phillips, and G. Caryotakis "Performance of a 150MW S-band Klystron", SLAC-PUB-6682, September 1994.

[22] H. Frischholz, W.R. Fowkes, and C. Pearson "Design and construction of a $500 \mathrm{~kW} \mathrm{CW}, 400 \mathrm{MHz}$ Klystron to be used as RF sources for LHC/RF component tests", Proceedings of the 1997 Particle Accelerator Conference, Vancouver, Canada, pp.2911-2913 May,1997.

[23] B. Krietenstein, T. Lee, U. Becker, T. Weiland, and M. Dohlus, "Spurious oscillations in high power Klystrons", in Proceedings Particle Accelerator Conference, Dallas, Texas, US, May 1995, doi: 10.1109/PAC.1995.505276.

[24] K.L.F. Bane, A. Jensen, Z. Li, G. Stupakov and C. Adolphsen, "Sheet beam Klystron instability analysis", in Proceedings Particle Accelerator Conference, Vancouver, BC, Canada, May 2009.

[25] K.W. Lee, J.G. Jeong, Y.J. Yoon, J.H. Kim, C.Kook, , “Analysis of the quality factor of input cavity with intense beam loading in a relativistic vacuum tube", IET Science, Measurement \& Technology, vol. 11 no. 2 pp. 141-148, March 2017, doi: 10.1049/iet-smt.2015.0298.

[26] Bruce E. Carlsten and Patrick Ferguson, "Numerical Determination of the Matching Conditions and Drive Characteristics for a Klystron Input Cavity with Beam", IEEE Trans. on Electron Devices, vol. 44, no. 5, pp. 894-900, May, 1997, doi:10.1109/16.568055.

[27] Craig B. Wilsen, John W. Luginsland, Yue Ying Lau, Thomas M. Antonsen, David P. Chernin, Philip M. Tchou, Marc W. Keyser, Ronald M. Gilgenbach, and L. D. Ludeking, "A Simulation Study of Beam Loading on a Cavity", IEEE Trans. on Plasma Science, vol. 30, no. 3, pp. 1160-1168, Jun., 2002, doi: 10.1109/TPS.2002.801623.

[28] R. Appleby, G. Burt, J. Clarke, H. Owen, The science and technology of particle accelerators. UK: CRC Press. December, 2020.

[29] J. J. Barroso, "Stepped electric-field profiles in transit-time tubes", IEEE Trans. Electron Devices, May, vol. 52 no. 5 pp. 872-877, May 2005 doi: 10.1109/T-ED.2005.845813. 